\title{
The imaging Maastricht Acute Stress Test (iMAST): A neuroimaging compatible psychophysiological stressor
}

Citation for published version (APA):

Quaedflieg, C. W. E. M., Meyer, T., \& Smeets, T. (2013). The imaging Maastricht Acute Stress Test (iMAST): A neuroimaging compatible psychophysiological stressor. Psychophysiology, 50(8), 758-766. https://doi.org/10.1111/psyp.12058

Document status and date:

Published: 01/08/2013

DOI:

10.1111/psyp. 12058

Document Version:

Publisher's PDF, also known as Version of record

Document license:

Taverne

Please check the document version of this publication:

- A submitted manuscript is the version of the article upon submission and before peer-review. There can be important differences between the submitted version and the official published version of record.

People interested in the research are advised to contact the author for the final version of the publication, or visit the DOI to the publisher's website.

- The final author version and the galley proof are versions of the publication after peer review.

- The final published version features the final layout of the paper including the volume, issue and page numbers.

Link to publication

\footnotetext{
General rights rights.

- You may freely distribute the URL identifying the publication in the public portal. please follow below link for the End User Agreement:

www.umlib.nl/taverne-license

Take down policy

If you believe that this document breaches copyright please contact us at:

repository@maastrichtuniversity.nl

providing details and we will investigate your claim.
}

Copyright and moral rights for the publications made accessible in the public portal are retained by the authors and/or other copyright owners and it is a condition of accessing publications that users recognise and abide by the legal requirements associated with these

- Users may download and print one copy of any publication from the public portal for the purpose of private study or research.

- You may not further distribute the material or use it for any profit-making activity or commercial gain

If the publication is distributed under the terms of Article $25 \mathrm{fa}$ of the Dutch Copyright Act, indicated by the "Taverne" license above, 


\title{
The imaging Maastricht Acute Stress Test (iMAST): A neuroimaging compatible psychophysiological stressor
}

\author{
CONNY W. E. M. QUAEDFLIEG, T. MEYER, AND T. SMEETS \\ Faculty of Psychology and Neuroscience, Maastricht University, Maastricht, The Netherlands
}

\begin{abstract}
Several protocols have been developed for inducing acute stress in laboratory settings. Still, effectively eliciting stress in a neuroimaging environment remains challenging. Here, we describe the evaluation of a combined physical and psychosocial stress protocol $(n=42)$. The imaging Maastricht Acute Stress Test (iMAST) consists of a 5-min preparation phase and a 10-min acute stress phase, with alternating trials of cold pressor stress generated through an advanced thermal stimulator and mental arithmetic challenges along with social-evaluative pressure (i.e., negative feedback). Results demonstrate that participants displayed meaningful subjective stress responses, as well as significant increases in salivary alpha-amylase and cortisol levels. Our data show that the iMAST may prove to be a potent alternative to existing imaging stress paradigms to explore poststress neuronal changes and brain determinants of resilience.
\end{abstract}

Descriptors: Cortisol, Functional magnetic resonance imaging (fMRI), Maastricht Acute Stress Test (MAST), Stress tasks, Stress reactivity

When a situation is perceived as a physical or psychological threat, the brain activates two physiological systems that jointly enable the individual to cope with the current stressor. The rapidly acting autonomic nervous system (ANS) ensures the release of adrenaline and noradrenaline, which help to potentiate arousal, alertness, and focused attention. In turn, the stimulation of the slower hypothalamic-pituitary-adrenal (HPA) axis, following a cascade of events, results in the secretion of the primary human glucocorticoid cortisol (Ulrich-Lai \& Herman, 2009). The contribution of various brain areas in regulating neuroendocrine stress responses depends on the severity and type of stress. Physical stressors (e.g., pain) are known to rapidly activate the ANS and HPA axis via the brainstem, whereas psychological stressors (e.g., social challenge tests) are mainly processed by the prefrontal cortex (PFC) and engage the limbic system (Dickerson \& Kemeny, 2004; Herman et al., 2003; Ulrich-Lai \& Herman, 2009).

Numerous protocols have been developed for inducing acute stress in laboratory settings, with the psychosocial Trier Social Stress Test (TSST; Kirschbaum, Pirke, \& Hellhammer, 1993) and the physical cold pressor test (CPT; Lovallo, 1975; Mitchell, MacDonald \& Brodie, 2004) being used most frequently. The TSST consists of a short preparation period followed by a speech

This research was supported by grants 451-08-005 and 056-25-011 from the Netherlands Organization for Scientific Research (NWO) to TS. NWO had no further role in the study design; in the collection, analysis and interpretation of the data; in the writing of the report; and in the decision to submit the paper for publication. We are especially grateful to Phyllis Mania for her help in collecting the data.

Address correspondence to: Conny W. E. M. Quaedflieg, Faculty of Psychology and Neuroscience, Maastricht University, P.O. Box 616, 6200 MD Maastricht, The Netherlands. E-mail: conny.quaedflieg@ maastrichtuniversity.nl and mental arithmetic task of 5 min each, both performed in front of an audience. In the CPT, participants are instructed to immerse their hand in ice-cold water for as long as possible for a maximum of 3 min. Both paradigms differ in the degree of ANS and HPA axis stimulation, which is related to different degrees of uncontrollability, unpredictability, and social evaluation (Dickerson \& Kemeny, 2004). The recently developed Maastricht Acute Stress Test (MAST) combines a physical (i.e., hand immersion in ice-cold water) and psychological (i.e., mental challenges including psychosocial evaluative threat) stressor, and has been shown to be a concise and valid procedure to elicit robust subjective, ANS, and HPA axis stress responses (Smeets et al., 2012).

In contrast to a range of laboratory stress induction procedures, effectively eliciting stress in a functional magnetic resonance imaging (fMRI) environment has proven to be exceedingly challenging. Until now, several psychological stress paradigms have been used, such as the Montreal Imaging Stress Task ((event)MIST; Dedovic et al., 2005; Dedovic, Rexroth et al., 2009), a serial subtraction task (i.e., subtracting 13 from a 4-digit number; see Wang et al., 2005, 2007), displaying emotional pictures (e.g., Sinha, Lacadie, Skudlarski, \& Wexler, 2004; Yang et al., 2007), or showing aversive movies (e.g., Cousijn, Rijpkema, Qin, van Wingen, \& Fernandez, 2012; Cousijn et al., 2010; Henckens, Hermans, Pu, Joels, \& Fernandez, 2009; Qin, Hermans, van Marle, Luo, \& Fernandez, 2009; van Marle, Hermans, Qin, \& Fernandez, 2009). In comparison to nonimaging laboratory stress paradigms like the TSST and MAST, the serial subtraction as well as the emotional stimuli paradigms result in relatively modest cortisol increases. Potential reasons for this may be relatively lower social evaluative threat and achievement demands (for a review of used stress tasks in a neuroimaging environment, see Dedovic, D'Aguiar, \& Pruessner, 2009). Moreover, until now, only one 
physically stressful paradigm, the CPT, has been used in fMRI studies. For example, Porcelli et al. (2008) investigated the effects of acute physical stress on working memory. Stress was induced by four 30-s hand-immersion trials in a bucket of $4{ }^{\circ} \mathrm{C}$ water. Of course, working with liquids in a scanning environment is cumbersome and far from ideal.

Paramount among the imaging stress tests so far is the (event)MIST, which is composed of a series of computerized mental arithmetic tasks with an induced failure algorithm and a social evaluative threat component (Dedovic et al., 2005; Dedovic, Rexroth et al., 2009). The (event)MIST thus incorporates key characteristics of psychological stress paradigms within the constraints of a neuroimaging environment and, compared to a control condition, elicits a significant hormonal (i.e., cortisol) stress response. Nevertheless, cortisol responses to the (event)MIST are significantly smaller than those of nonimaging stress protocols, and there is a large variation between studies applying the (event)MIST in the number of cortisol responders as well as the maximal cortisol increase (see, e.g., Dagher, Tannenbaum, Hayashi, Pruessner, \& McBride, 2009; Dedovic et al., 2005; Dedovic, Rexroth et al., 2009; Khalili-Mahani, Dedovic, Engert, Pruessner, \& Pruessner, 2010; Lederbogen et al., 2011; Lord, Steiner, Soares, Carew, \& Hall, 2012; Pruessner et al., 2008). This variation may be due to the switching between experimental, rest, and control conditions in the (event)MIST, which might not be optimal for the induction of stress in the majority of people.

The current study was set out to develop and evaluate a stress protocol that is effective in eliciting robust cortisol and alphaamylase responses in an fMRI environment, which would provide a reliable means for investigating poststress neuronal changes. We adapted the MAST (Smeets et al., 2012) to create an fMRIcompatible, physically and psychologically challenging stress test, which is labeled the imaging Maastricht Acute Stress Test (iMAST). Basically, and closely mimicking the MAST, the iMAST consists of a 5-min preparation phase in which task instructions are given and a 10-min acute stress phase. The acute stress phase includes several exposures to cold pressor stress generated with an advanced thermal stimulator (ATS) and various mental arithmetic challenges along with social-evaluative pressure (i.e., negative feedback). It was hypothesized that the iMAST would induce subjective stress and would result in significant increases in salivary alpha-amylase and cortisol.

\section{Method}

\section{Participants}

Forty-two right-handed scanner-naïve participants (21 men, 21 women, mean age $=21.8$ years, $S D=2.14$; range: $18-35$ ) participated in the current study. All subjects underwent a screening protocol assessing physical and mental health, fMRI aptness, and handedness. Participants were excluded when they had a history of psychiatric, neurologic, cardiovascular, or neuroendocrine disease, were considered heavy smokers (i.e., more than 15 cigarettes/day), used medication known to affect the ANS or HPA axis, were regular drug users, or had a body mass index (weight in $\mathrm{kg}$ divided by (height in $\mathrm{m})^{2}$ ) outside the $18-30$ range. An additional exclusion criterion was prior experience with the standard (i.e., nonimaging) MAST. Moreover, for women, an extra inclusion criterion was the use of oral contraceptives to reduce variability in cortisol responses related to hormonal alterations throughout the menstrual cycle phase (e.g., Kudielka, Hellhammer, \& Wust, 2009). All types of oral contraceptives were allowed, and the intake phase was not controlled for. Pregnancy or lactation, on the other hand, served as additional exclusion criteria for women. Test protocols were approved by the standing Ethics Committee of the Faculty of Psychology and Neuroscience, Maastricht University. All participants signed a written informed consent and were given a small monetary reward in return for their participation.

\section{Stress Induction Equipment and Procedures}

An imaging-compatible version of the MAST (Smeets et al., 2012) was used to induce stress in the fMRI scanner. The iMAST consists of a 5-min preparation phase in which the task is explained and a 10-min acute stress phase, which includes several exposures to cold pressor stress and various mental arithmetic challenges along with social-evaluative pressure (i.e., negative feedback). Notably, in laboratory studies, cold pressor stress is induced by asking participants to submerge their hand in ice-cold water, yet this is not suitable for a scanning environment. Therefore, cold pressor stress in the iMAST was generated with an fMRI compatible $30 \times 30 \mathrm{~mm}$ Medoc Pathway ATS thermode (Medoc Ltd., Ramat Yishai, Israel) placed on the left volar forearm. In total, five cold pressor stress stimuli of $2{ }^{\circ} \mathrm{C}$ were applied with a variable duration of 45,60 , or $90 \mathrm{~s}$. In between the cold pressor trials, participants had to engage in mental arithmetic challenges, that is, counting backwards as fast and accurately as possible in steps of 17 starting at 2,043 for 45, 60, or $90 \mathrm{~s}$ (see Figure 1). The participants were equipped with a magnetcompatible headset, consisting of earphones and a microphone that was attached to the headphone. The experimenter monitored the counting via the intercom system and, whenever participants counted too slowly or made a mistake, they received negative feedback via the intercom (i.e., to count faster or start over at 2,043). During these mental arithmetic trials, the baseline temperature of the ATS was $25^{\circ} \mathrm{C}$. Additionally, during each of the mental arithmetic trials, one, two or three hot pulses $\left(49^{\circ} \mathrm{C}\right)$ of $10 \mathrm{~s}$ occurred without warning to increase the unpredictability of the task. Moreover, similar to the standard MAST, the iMAST protocol sought to increase uncontrollability and unpredictability by telling participants that the computer would randomly choose the order and duration of the cold pressor and mental arithmetic trials. However, in reality, the duration and order of cold pressor stress stimuli and arithmetic trials was fixed for all participants (see Figure 1). During stress induction, high resolution, T1-weighted structural images of the whole brain were acquired using a 3 Tesla Siemens MR headonly scanner (MAGNETOM Allegra, Siemens Medical Systems, Erlangen, Germany). The imaging data will be addressed elsewhere.

\section{Subjective and Neuroendocrine Stress Responses}

Subjective stress. Three $100-\mathrm{mm}$ visual analogue scales (VAS) were used to assess subjective stress effects related to the iMAST. After the scanning session had ended, participants had to specify their level of agreement with statements on how stressful, painful, and unpleasant they felt during the iMAST by indexing the VAS scales (anchors: $0=$ not at all, $100=$ extremely).

Salivary alpha-amylase (sAA) and cortisol. Neuroendocrine stress measures prior to and in response to the iMAST were obtained with synthetic Salivette (Sarstedt, Etten-Leur, The Netherlands) devices. Participants provided saliva samples immediately after having been placed in the fMRI scanner $\left(t_{M R I}\right), 5$ min before the iMAST ( $t_{\text {pre-stress }}$; i.e., $25 \mathrm{~min}$ after the $t_{\text {MRI }}$ sample was taken) 


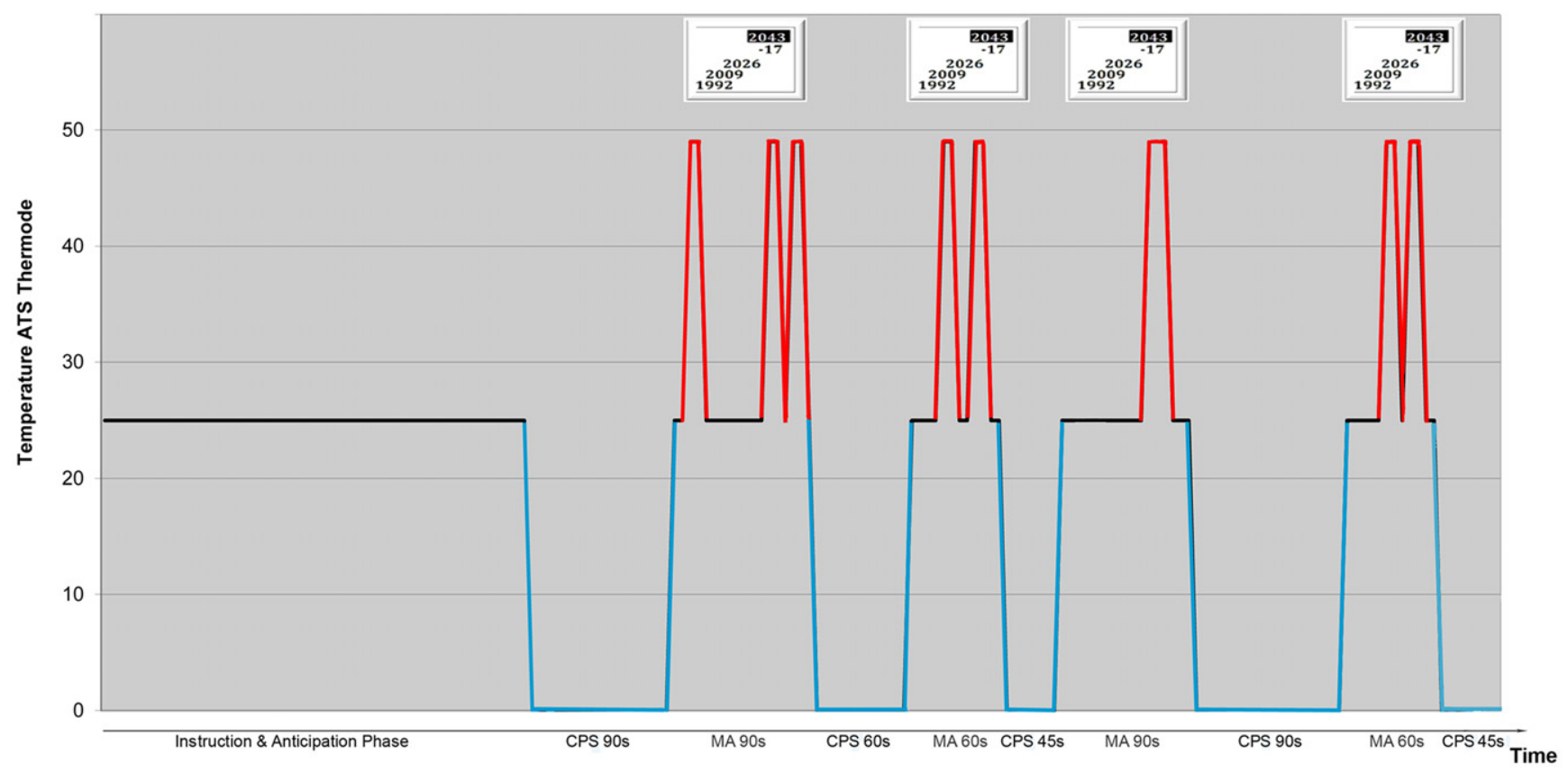

Figure 1. Order, duration (in seconds), and temperature of the cold pressor stress (CPS) and mental arithmetic (MA) trials of the iMAST.

and four times afterwards $\left(t_{+0}, t_{+10}, t_{+30}, t_{+40}\right.$ with reference to the end of the stressor). The timing of saliva sampling was based on previous work showing that sAA peaks immediately after stress onset (e.g., Nater et al., 2005; Rohleder, Nater, Wolf, Ehlert, \& Kirschbaum, 2004), while cortisol typically peaks 20 to $40 \mathrm{~min}$ after stress onset (e.g., Dickerson \& Kemeny, 2004). For each sample collection, participants were returned to the home position of the scanner, and a research assistant then placed the Salivette in the mouth of the participant using sterile plastic tweezers. To facilitate sample collection and to minimize movement, this procedure was practiced during a simulation scan in a dummy MRI device beforehand. Saliva samples were stored at $-20^{\circ} \mathrm{C}$ immediately on collection. sAA and cortisol levels were determined from the saliva samples using a commercially available kinetic reaction assay (Salimetrics, Penn State, PA) and luminescence immune assay kit (IBL, Hamburg, Germany), respectively. Mean intra- and interassay coefficients of variation are typically less than $8 \%$ and $6 \%$ for the sAA analyses and less than $5 \%$ for both for the cortisol analyses.

\section{Study Procedures}

All testing took place between 12:30 and 18:00 to control for the circadian rhythm of cortisol (Nicolson, 2008). Participants were asked to refrain from eating, exercising extensively, or drinking anything but nonsparkling water for $2 \mathrm{~h}$ prior to the experimental session. After arrival in the laboratory, participants received information on the experimental procedure and gave written informed consent. Next, a saliva sample was taken with the explicit but bogus instruction that it would be immediately assayed to check whether they had adhered to our instructions not to eat, drink, etc. In actual fact, the sample was destroyed without being analyzed. This procedure was followed to promote truth-telling behavior when participants were subsequently asked whether they had adhered to the instructions. For each measurement, participants were instructed to place the Salivette on the same side of their mouth and not to chew on it (see also Beltzer et al., 2010). Next, participants received a standardized lunch (a sandwich and 0.51 of nonsparkling water) during which they were required to fill out several questionnaires (data not reported here). Thereafter and for purposes unrelated to the aims of the current report, electroencephalography (EEG) measurement was prepared and resting EEG activity was measured during $8 \mathrm{~min}$ (data to be reported elsewhere). One and a half hours after arrival, participants were prepared for a 30-min simulation scan to familiarize them with the environment and to reduce potential scanner-induced stress effects (e.g., Muehlhan, Lueken, Wittchen, \& Kirschbaum, 2011). We used a dummy MRI device with exactly the same appearance and noise (transmitted via headphones) as the fMRI scanner in order to mimic the actual fMRI session as closely as possible. Moreover, sAA/cortisol sampling and counting aloud was practiced to minimize head movement during saliva sampling and the iMAST in the scanner. The experimenter observed the participants during training in the dummy scanner and decided whether and when the movement was so insignificant that it was acceptable to start with the actual scanning. Subsequently, participants were placed in the 3 Tesla Siemens MR head-only scanner (MAGNETOM Allegra) and a baseline sAA/ cortisol sample ( $t_{\text {MRI }}$ ) was taken. The fMRI session lasted for about $1.5 \mathrm{~h}$ and consisted of the following runs (in chronological order): resting state before stress induction (duration: $8 \mathrm{~min}$ ), baseline emotional reactivity (16 min), iMAST including anatomical scan (15 min), resting state after stress induction $(8 \mathrm{~min})$, emotional working memory task (18 $\mathrm{min})$, and recovery resting state (8 min). In total, six saliva samples were collected during scanning (see above). After completion of the scanner session, participants completed some questionnaires including the VAS items about the iMAST and provided ratings of the stimuli used in the emotional reactivity task (see Figure 2 for an overview of the design). 


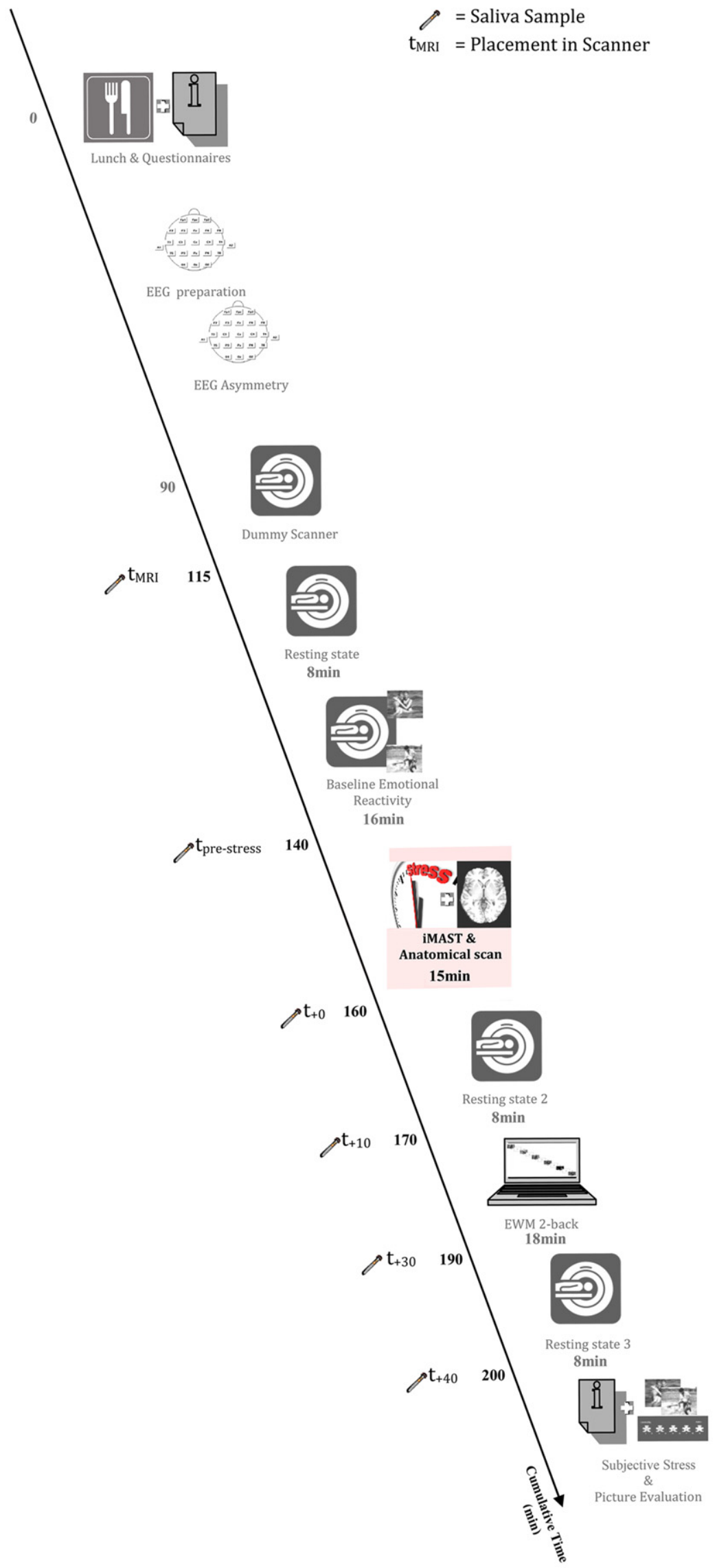

Figure 2. Overview of the design and exact timing of the saliva samples. 
Table 1. Means ( \pm SE) of Subjective Stress for Male and Female Participants

\begin{tabular}{lcc}
\hline \hline & Male & Female \\
\hline Subjective stress (0-100) & & \\
Stress & $76.67(4.29)$ & $71.81(4.49)$ \\
Pain & $53.05(6.12)$ & $54.76(4.89)$ \\
Unpleasantness & $71.71(4.75)$ & $75.00(5.02)$ \\
\hline \hline
\end{tabular}

\section{Data Analysis}

Descriptive statistics for subjective stress ratings (VAS scales) are reported, and differences in ratings between men and women were analyzed using univariate analyses of variance (ANOVAs). Cortisol and sAA data were log-transformed before analyses as ShapiroWilk tests of normality showed typical skewness of the data. Cortisol and sAA responses were analyzed using a repeated measures ANOVA with time ( 6 levels: $\left.t_{\text {MRI }}, t_{\text {pre-stress }}, t_{+0}, t_{+10}, t_{+30}, t_{+40}\right)$ as within-subject factor and gender as between-subjects factor. For each participant individually, the area under the curve with respect to increase (AUCi) was calculated as a single measure of the total hormone (i.e., sAA and cortisol) concentration in response to the iMAST (Pruessner, Kirschbaum, Meinlschmid, \& Hellhammer, 2003). The delta increase in cortisol was also computed, defined as peak cortisol concentration after the iMAST minus prestress cortisol concentration. Moreover, a responder rate was calculated representing participants with a cortisol increase equal to or larger than $2.5 \mathrm{nmol} / \mathrm{l}$ (see, for example, Kirschbaum et al., 1993; Smeets, Jelicic, \& Merckelbach, 2006), which is thought to reflect a cortisol secretory episode (Van Cauter \& Refetoff, 1985). Descriptive statistics for all measures are reported, and differences between men and women were analyzed using chi-square tests and univariate ANOVAs. Two male participants who did not provide enough saliva to be analyzed and one clear cortisol outlier (>3SD above the mean cortisol increase) were excluded from all analyses (i.e., for the cortisol analyses, the total $n=39$ ). Statistical effects were evaluated using the Greenhouse-Geisser correction and Bonferroni correction when appropriate. $P$-values smaller than .05 were considered statistically significant. In case of significant results, ANOVAs are supplemented with partial eta squared $\left(\eta_{p}^{2}\right)$ values as a measure of effect size $\left(\eta_{\mathrm{p}}^{2}\right.$ of 0.01 indicate small effects, $\eta_{\mathrm{p}}^{2}$ of 0.06 medium effects, and $\eta_{\mathrm{p}}^{2}$ of 0.14 large effects; Fritz, Morris, \& Richler 2012), and significant follow-up comparisons are supplemented with the standardized mean-change statistic $d$ ( $d$ of 0.20 indicate small effects, $d$ of 0.50 medium effects, and $d$ of 0.80 large effects; Dickerson \& Kemeny, 2004).

\section{Results}

\section{Subjective Stress Responses}

As shown in Table 1, subjects perceived the iMAST as distressing, as indicated by their ratings of subjective stress, pain, and unpleasantness. Male and female participants did not differ in their subjective reactions to the iMAST, as evidenced by nonsignificant main effects of gender (stressfulness: $F(1,40)=1.02, p=.32$; painfulness: $F(1,40)=.05, p=.83$; and unpleasantness: $F(1,40)=.09$, $p=.77)$.

\section{Neuroendocrine Stress Responses}

Mean salivary sAA/cortisol concentrations prior to and following the iMAST are shown in Figure 3. For the entire sample, ANOVAs pertaining to sAA data did not reveal a significant Time $\times$ Gender interaction (sAA: $F(3.69,95.98)=.81, p=.52$ ) but did yield the anticipated significant main effect of time (sAA: $\left.F(3.71,100.19)=5.34, p=.001 ; \eta_{\mathrm{p}}^{2}=0.16\right)$. Bonferroni corrected pairwise comparisons between adjacent time points were used to further evaluate the autonomic stress response to the iMAST over time. Comparison of the saliva sample at placement $\left(t_{\mathrm{MRI}}\right)$ with the sample immediately before the iMAST $\left(\mathrm{t}_{\text {pre-stress }}\right)$ demonstrated that lying in the scanner did not induce any changes in sAA levels $\left(p>\right.$.99). Significant increases in sAA were found between $t_{\text {pre-stress }}$ and $\mathrm{t}_{+0}(p=.007 ; d=0.62)$, followed by a decrease between $\mathrm{t}_{+0}$ and $\mathrm{t}_{+10}(p=.01 ; d=-0.46)$, only to remain stable afterwards (i.e., between $\mathrm{t}_{+10}$ vs. $\mathrm{t}_{+30}$ and $\mathrm{t}_{+30}$ vs. $\mathrm{t}_{+40}$; both $p \mathrm{~s}>.20$ ). The overall total sAA concentration (AUCi) in response to the iMAST was 857.70 (SEM 198.50) (females: $M=686.90, \quad S E M$ 141.63; males $M=1099.67, S E M$ 438.13). There were no differences in AUCi between males and females, $F(1,28)=1.05, p=.31$.

As to the cortisol data for the total sample, ANOVAs did not reveal a significant Time $\times$ Gender interaction (cortisol:
A

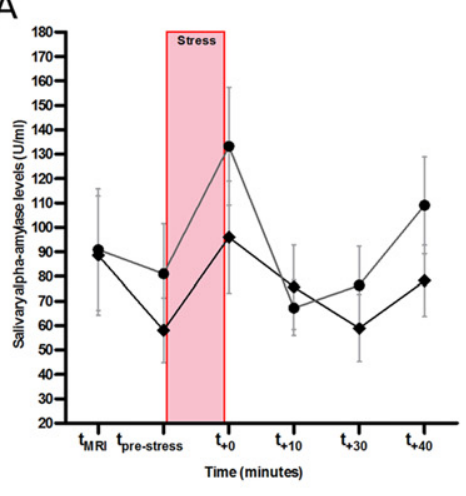

B

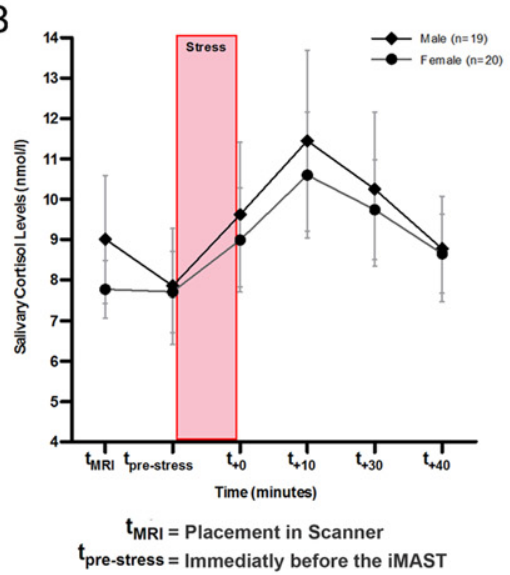

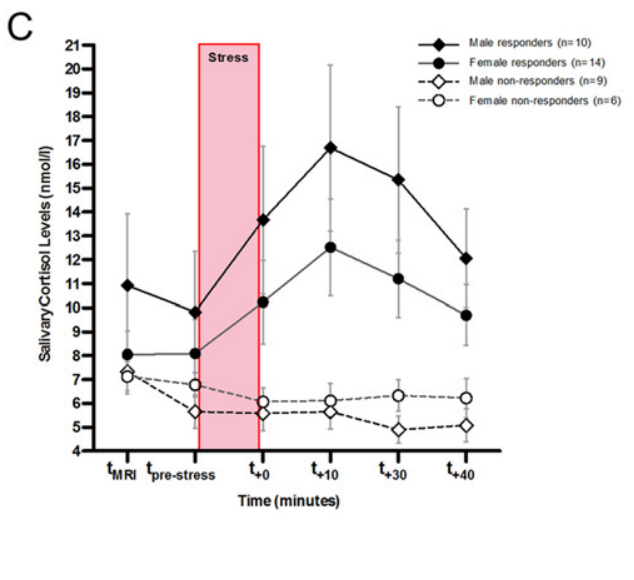

C

Figure 3. Salivary alpha-amylase and cortisol responses to the imaging Maastricht Acute Stress Test (iMAST) for male and female participants (A, B), and for male and female cortisol responders and nonresponders separately (C). Values represent (untransformed) means $\pm S E$. 
$F(2.30,82.68)=.20, p=.85)$ but again did reveal the expected significant main effect of time (cortisol: $F(2.30,85.04)=4.94$, $p=.007 ; \eta_{\mathrm{p}}^{2}=0.12$ ). Comparison of the saliva sample at placement ( $\left.t_{\text {MRI }}\right)$ with the sample immediately before the iMAST ( $\left.t_{\text {pre-stress }}\right)$ demonstrated that merely being inside the scanner did not result in a cortisol increase $(p>.99)$. Follow-up tests did show significant increases in cortisol between $\mathrm{t}_{\text {pre-stress }}$ and $\mathrm{t}_{+0}(p=.029 ; d=0.29)$ followed by a further increase in cortisol between $t_{+0}$ and $t_{+10}$ ( $p=.004 ; d=0.25$ ), only to remain stable afterwards (i.e., between $\mathrm{t}_{+10}$ vs. $\mathrm{t}_{+30}$ and $\mathrm{t}_{+30}$ vs. $\mathrm{t}_{+40}$; both $p \mathrm{~s}>.20$ ). Furthermore, relative to the prestress sample, cortisol remained significantly increased up until $\mathrm{t}_{+40}\left(\mathrm{t}_{\text {pre-stress }}\right.$ vs. $\mathrm{t}_{+10}, p<.001 ; d=0.61 ; \mathrm{t}_{\text {pre-stress }}$ vs. $\mathrm{t}_{+30}, p=.04$; $d=0.42$; $\mathrm{t}_{\text {pre-stress }}$ vs. $\left.\mathrm{t}_{+40}, p>.99\right)$. The overall total cortisol concentration (AUCi) in response to the iMAST was 112.92, SEM 22.90 (females: $M=104.46$, SEM 29.78; males $M=120.99$, SEM 35.19). There were no differences in AUCi between males and females, $F(1,40)=0.13, p=.72$.

We then proceeded by calculating a responder rate, representing participants with a cortisol increase equal to or larger than $2.5 \mathrm{nmol} / \mathrm{l}$, which is thought to reflect a cortisol secretory episode. The percentage of participants who could be classified as cortisol responders was $61.5 \%$ (female: $M=70 \%$; male $M=53 \%$ ) and did not differ by gender, $\chi^{2}(1, N=39)=1.24, p=.27$.

Delta increases in cortisol, that is, peak cortisol concentration after the iMAST minus prestress cortisol concentration, were also computed. The overall mean cortisol increase (i.e., delta cortisol) was $3.78 \mathrm{nmol} / 1$ (SEM 0.64) (female: $M=3.10$, SEM 0.65; male $M=4.47, S E M 1.10)$. For the cortisol responder group, the mean cortisol increase was $7.90 \mathrm{nmol} / 1$ (SEM 1.30) for males while the mean increase for females was $4.54 \mathrm{nmol} / \mathrm{l}$ (SEM 0.60). For the cortisol responder group, univariate ANOVA on delta cortisol responses indicated a main effect of gender, $F(1,21)=6.45, p=.02$ $\eta_{\mathrm{p}}^{2}=0.24$. In contrast, no gender differences with respect to delta cortisol increase were found among the cortisol nonresponders, $F(1,13)=1.21, p=.29$; delta cortisol female: $M=-.02$ (SEM 0.38 ); male $M=.66$ (SEM 0.43).

\section{Discussion}

The primary aim of the current study was to evaluate the effectiveness of the iMAST as a stress induction paradigm that is capable of eliciting subjective, autonomic, and HPA axis stress responses within the constraints of a neuroimaging environment. Results demonstrate that the iMAST succeeded in generating considerable subjective stress responses in terms of stressfulness, painfulness, and unpleasantness, as well as robust increases in sAA and cortisol levels in the majority of participants.

The iMAST is the first neuroimaging-suited stress task that relies on a procedure comparable to classic behavioral laboratory stress tasks and thus is characterized by unpredictability and uncontrollability. Additionally, it includes a physical component designed to elicit an immediate bodily reaction via reflexive mechanisms in the brainstem and hypothalamus, resulting in a rapid activation of the ANS and HPA axis (Ulrich-Lai \& Herman, 2009). Moreover, the explicit monitoring of and verbal negative feedback on participants' performance while in the scanner creates a social evaluative threat component that triggers stress responses of the HPA axis, mediated by an activation of the PFC, thalamus, and limbic structures (Herman et al., 2003; Ulrich-Lai \& Herman, 2009). Thus, similar to other laboratory stress tasks, the iMAST follows a double route to stimulate the HPA axis, which makes it possible to evaluate the contribution of different brain areas in poststress regulation of cognitive and physiological processes.

Further attesting to the effectiveness of the iMAST in activating the HPA axis, we found that $61.5 \%$ of all participants displayed cortisol increases larger than $2.5 \mathrm{nmol} / \mathrm{l}$ (indicative of a cortisol secretory episode; Van Cauter \& Refetoff, 1985) and could be reliably classified as cortisol responders. Note that this is a more conservative and strict definition of what counts as a cortisol responder, compared to previous neuroimaging studies that employed a post hoc responder classification of participants who displayed cortisol increases that were much smaller than $2.5 \mathrm{nmol} / 1$ (e.g., Dedovic, Rexroth et al., 2009; Khalili-Mahani et al., 2010; Lederbogen et al., 2011; Pruessner et al., 2008; Soliman et al., 2011). While still not approximating some of the most potent nonimaging stress tests such as the TSST and MAST, the current results show that the iMAST is capable of generating significant cortisol responses that are comparable to some other nonimaging laboratory stress tests (e.g., CPT, socially evaluated cold pressor test [SECPT]; e.g., Schwabe, Haddad, \& Schachinger, 2008; Schwabe \& Wolf, 2010; Smeets et al., 2012, Study 1; Hupbach \& Fieman, 2012) and may even exceed those of the existing neuroimaging-suited stress tasks such as the (event)MIST (Dedovic et al., 2005; Dedovic, Rexroth et al., 2009).

Notably, we showed that the fMRI procedure by and of itself did not induce significant sAA or cortisol changes, which may be due to the preparation for the MRI scanning procedure including a practice trial in a dummy MRI device. This then, to some extent, disagrees with previous work suggesting that the anticipation of fMRI measurements can moderately increase subjective (Dantendorfer et al., 1997; but see Tessner, Walker, Hochman, \& Hamann, 2006) and neuroendocrine stress markers (Muehlhan et al., 2011; Tessner et al., 2006; van Stegeren, Rohleder, Everaerd, \& Wolf, 2006), even when a dummy scan preceded the actual MRI measurements (Eatough, Shirtcliff, Hanson, \& Pollak, 2009; Lueken, Muehlhan, Evens, Wittchen, \& Kirschbaum, 2012). These conflicting findings may be related to differences in the timing of the sampling procedure (e.g., Kukolja, Thiel, Wolf, \& Fink, 2008), population characteristics (e.g., age, gender), or the design (e.g., two separate scanning sessions). Additionally, Lueken et al. (2012) proposed that the reassuring influence of dummy scanner training depends on the physical arrangement and instructions given. In order to abolish anticipatory anxiety and familiarize them with the scanning environment, participants in the current study were extensively trained in the dummy scanner. This training session lasted for about $30 \mathrm{~min}$ and included general information about fMRI measurements, required participants to touch the outside of the scanner while actually being inside so as to get a feeling of their own position, and simulated the noise of the MRI scanner.

Note that the current study included a stress, but not a no-stress control condition. Admittedly, to further investigate the stresseliciting effects of the scanner environment (e.g., Muehlhan et al., 2011), and for future research that aims to investigate the effects of stress on various (e.g., cognitive) tasks more broadly, a no-stress placebo version of the iMAST is needed. Consistent with the placebo version for the MAST (see Smeets et al., 2012; Study 3), the iMAST's no-stress placebo version could consist of small, relatively neutral (e.g., $35^{\circ} \mathrm{C}$ and $40^{\circ} \mathrm{C}$ ) deviations from the normal body temperature, and a simplified counting task (counting consecutively from 1 to 25) without performance feedback. This way, the no-stress placebo version would be similar to the iMAST in 
terms of the order of trials and its duration, but without eliciting stress reactions.

It is well known that laboratory stressors may generate sex differences in HPA axis responses, with typically larger increases found in males compared to females (Kirschbaum, Wust, \& Hellhammer, 1992; for review, see Kajantie \& Phillips, 2006; Kudielka et al., 2009; Kudielka \& Kirschbaum, 2005), which was confirmed in the current study. Indeed, psychophysiological studies suggest a role for hormonal activity that is specifically related to the menstrual cycle (e.g., Kirschbaum, Kudielka, Gaab, Schommer, \& Hellhammer, 1999) and its associated differential activation of subcortical arousal structures that are part of stress response circuitry (e.g., Goldstein, Jerram, Abbs, Whitfield-Gabrieli, \& Makris, 2010). For instance, Wang et al., 2007, found that stress activated the right prefrontal cortex in men, whereas in women stress activated the ventral striatum, putamen, insula, and cingulate cortex. Notably, these differences were observed in the absence of differences in physiological response between genders. Moreover, Wang and colleagues (2007) also showed that, after completion of the serial subtraction task, only women displayed activity in the anterior and posterior cingulate cortex, structures that have been implicated in emotional processing and reflection of emotional traits. Both lines of research indicate quantitative and qualitative gender differences in stress regulation and indicate the need for combining neuroimaging, physiological, and behavioral approaches.

Even though the iMAST results in robust subjective and neuroendocrine (e.g., cortisol) stress responses, there may also be some potential drawbacks to using the iMAST to generate stress in an fMRI environment. For instance, the iMAST needs the appropriate additional equipment (e.g., the Medoc Pathway stimulator and ATS thermode). Moreover, the physical component of the iMAST might be too intense for certain samples (i.e., children, clinical groups). On the other hand, by having added this physical component to the iMAST, an immediate bodily reaction via reflexive mechanisms implicating the brainstem and hypothalamus (Herman et al., 2003; Ulrich-Lai \& Herman, 2009) was ensured, thus triggering a rapid activation of the ANS and the HPA axis, which in combination with the psychosocial stress components may have been the reason why the iMAST has proven to be effective in yielding solid stress responses. Also, the iMAST requires meticulous training (e.g., in a dummy scanner) beforehand to reduce or eliminate head movement during the counting test. To this end, we instructed participants not to be concerned with their own audibility, and, additionally, foam padding was placed around the subject's head in the head coil. It is worth mentioning here that many other studies also used speech during neuroimaging. For example, research investigating the semantic interference effect uses the picture naming task in which participants have to label pictures aloud. These studies looked specifically at the movement induced by speech, and reported less than 3-mm head movement (e.g., Barch, Braver, Sabb, \& Noll, 2000; Christoffels, Formisano,
\& Schiller, 2007; Christoffels, van de Ven, Waldorp, Formisano, \& Schiller, 2011; Kan \& Thompson-Schill, 2004; Shuster \& Lemieux, 2005). Besides, rotational and translational movement less than the voxel size can be handled well by motion correction. Although we do not have movement parameters from the dummy training or during the anatomical scan, the translational and rotational parameters during the resting state functional runs indicate that all participants moved less than $2 \mathrm{~mm}$. Finally, the current design of the iMAST only allows for investigating the poststress contribution of different brain areas during stress regulation. That is, other imaging stress tests, such as the (event)MIST and serial subtraction, may be more suitable for investigating neural processes during stress induction. Note, however, that the aim of the current study was to develop and evaluate a stress paradigm that closely resembled standard behavioral laboratory stress tests and procedures (e.g., TSST, MAST) that typically investigate effects of stress on cognitive processes by administering a task after stress induction.

A few limitations that are specific to the current study also deserve to be mentioned. First, as is typical for research employing VAS scales to assess subjective stress, ratings were obtained after the completion of the stress induction protocol only. However, as a recent study by Hellhammer and Schubert (2012) showed that subjective stress ratings during but not before or after the stress task are associated with physiological stress parameters, repeatedly assessing subjective stress, especially during the iMAST, could prove to be valuable. Second, with respect to gender differences, it may be important to note that all women used oral contraceptives. We used this inclusion criterion to avoid cortisol response variation related to the female menstrual cycle (Kirschbaum et al., 1999; Kudielka et al., 2009). However, since the hormonal activity of the female menstrual cycle may modulate the activation of the stress response circuitry (Goldstein et al., 2010), future studies may opt to include women during the various phases of the menstrual cycle, which would provide a unique opportunity to investigate the impact of gender-specific hormones. That way, one could also establish whether, in line with laboratory stress paradigms such as the TSST, cortisol responses to the iMAST are comparable for men and naturally cycling women in their late luteal phase (Kirschbaum et al., 1999).

In sum, the current study demonstrates the value of the iMAST as a concise stress paradigm capable of reliably eliciting strong subjective, autonomic, and glucocorticoid stress responses in a neuroimaging environment. Our data show that the iMAST may prove to be a powerful alternative to other paradigms in research that seeks a combination of a physical and psychosocial stressor. The iMAST presents researchers with new opportunities for investigating the effects of combined physical and psychological stressors that may aid in the exploration of the anatomical and functional connectivity of the human brain as determinants of individuals' degree of stress resilience or vulnerability.

\section{References}

Barch, D. M., Braver, T. S., Sabb, F. W., \& Noll, D. C. (2000). Anterior cingulate and the monitoring of response conflict: Evidence from an fMRI study of overt verb generation. Journal of Cognitive Neuroscience, 12, 298-309. doi: 10.1162/089892900562110

Beltzer, E. K., Fortunato, C. K., Guaderrama, M. M., Peckins, M. K., Garramone, B. M., \& Granger, D. A. (2010). Salivary flow and alpha-amylase: Collection technique, duration, and oral fluid type. Physiology \& Behavior, 101, 289-296. doi: 10.1016/j.physbeh.2010. 05.016
Christoffels, I. K., Formisano, E., \& Schiller, N. O. (2007). Neural correlates of verbal feedback processing: An fMRl study employing overt speech. Human Brain Mapping, 28, 868-879. doi: 10.1002/Hbm.20315 Christoffels, I. K., van de Ven, V., Waldorp, L. J., Formisano, E., \& Schiller, N. O. (2011). The sensory consequences of speaking: Parametric neural cancellation during speech in auditory cortex. PLoS One, 6, e18307. doi: 10.1371/journal.pone.0018307

Cousijn, H., Rijpkema, M., Qin, S. Z., van Marle, H. J. F., Franke, B., Hermans, E. J., ... Fernandez, G. (2010). Acute stress modulates 
genotype effects on amygdala processing in humans. Proceedings of the National Academy of Sciences of the United States of America, 107, 9867-9872. doi: 10.1073/pnas.1003514107

Cousijn, H., Rijpkema, M., Qin, S. Z., van Wingen, G. A., \& Fernandez, G. (2012). Phasic deactivation of the medial temporal lobe enables working memory processing under stress. Neuroimage, 59, 1161-1167. doi: 10.1016/j.neuroimage.2011.09.027

Dagher, A., Tannenbaum, B., Hayashi, T., Pruessner, J. C., \& McBride, D. (2009). An acute psychosocial stress enhances the neural response to smoking cues. Brain Research, 1293, 40-48. doi: 10.1016/j.brainres. 2009.07.048

Dantendorfer, K., Amering, M., Bankier, A., Helbich, T., Prayer, D., Youssefzadeh, S., . . . Katschnig, H. (1997). A study of the effects of patient anxiety, perceptions and equipment on motion artifacts in magnetic resonance imaging. Magnetic Resonance Imaging, 15, 301306.

Dedovic, K., D’Aguiar, C., \& Pruessner, J. C. (2009). What stress does to your brain: A review of neuroimaging studies. Canadian Journal of Psychiatry-Revue Canadienne De Psychiatrie, 54, 6-15.

Dedovic, K., Renwick, R., Mahani, N. K., Engert, V., Lupien, S. J., \& Pruessner, J. C. (2005). The Montreal Imaging Stress Task: Using functional imaging to investigate the effects of perceiving and processing psychosocial stress in the human brain. Journal of Psychiatry \& Neuroscience, 30, 319-325.

Dedovic, K., Rexroth, M., Wolff, E., Duchesne, A., Scherling, C., Beaudry, T., . . Pruessner, J. C. (2009). Neural correlates of processing stressful information: An event-related fMRI study. Brain Research, 1293, 49-60. doi: 10.1016/j.brainres.2009.06.044

Dickerson, S. S., \& Kemeny, M. E. (2004). Acute stressors and cortisol responses: A theoretical integration and synthesis of laboratory research. Psychological Bulletin, 130, 355-391. doi: 10.1037/00332909.130.3.355

Eatough, E. M., Shirtcliff, E. A., Hanson, J. L., \& Pollak, S. D. (2009). Hormonal reactivity to MRI scanning in adolescents. Psychoneuroendocrinology, 34, 1242-1246. doi: 10.1016/j.psyneuen.2009.03.006

Fritz, C. O., Morris, P. E., \& Richler, J. J. (2012). Effect size estimates: Current use, calculations, and interpretation. Journal of Experimental Psychology: General, 141, 2-18. doi: 10.1037/a0024338

Goldstein, J. M., Jerram, M., Abbs, B., Whitfield-Gabrieli, S., \& Makris, N. (2010). Sex differences in stress response circuitry activation dependent on female hormonal cycle. The Journal of Neuroscience, 30, 431-438. doi: 10.1523/JNEUROSCI.3021-09.2010

Hellhammer, J., \& Schubert, M. (2012). The physiological response to Trier Social Stress Test relates to subjective measures of stress during but not before or after the test. Psychoneuroendocrinology, 37, 119-124. doi: 10.1016/j.psyneuen.2011.05.012

Henckens, M. J. A. G., Hermans, E. J., Pu, Z. W., Joels, M., \& Fernandez, G. N. (2009). Stressed memories: How acute stress affects memory formation in humans. Journal of Neuroscience, 29, 10111-10119. doi: 10.1523/Jneurosci.1184-09.2009

Herman, J. P., Figueiredo, H., Mueller, N. K., Ulrich-Lai, Y., Ostrander, M. M., Choi, D. C., \& Cullinan, W. E. (2003). Central mechanisms of stress integration: Hierarchical circuitry controlling hypothalamo-pituitaryadrenocortical responsiveness. Frontiers in Neuroendocrinology, 24, 151-180. doi: 10.1016/j.yfrne.2003.07.001

Hupbach, A., \& Fieman, R. (2012). Moderate stress enhances immediate and delayed retrieval of educationally relevant material in healthy young men. Behavioral Neuroscience, 126, 819-825. doi: 10.1037/a0030489

Kajantie, E., \& Phillips, D. I. (2006). The effects of sex and hormonal status on the physiological response to acute psychosocial stress. Psychoneuroendocrinology, 31, 151-178. doi: 10.1016/j.psyneuen. 2005.07.002

Kan, I. P., \& Thompson-Schill, S. L. (2004). Effect of name agreement on prefrontal activity during overt and covert picture naming. Cognitive, Affective, and Behavioral Neuroscience, 4, 43-57.

Khalili-Mahani, N., Dedovic, K., Engert, V., Pruessner, M., \& Pruessner, J. C. (2010). Hippocampal activation during a cognitive task is associated with subsequent neuroendocrine and cognitive responses to psychological stress. Hippocampus, 20, 323-334. doi: 10.1002/Hipo.20623

Kirschbaum, C., Kudielka, B. M., Gaab, J., Schommer, N. C., \& Hellhammer, D. H. (1999). Impact of gender, menstrual cycle phase, and oral contraceptives on the activity of the hypothalamus-pituitary-adrenal axis. Psychosomatic Medicine, 61, 154-162.

Kirschbaum, C., Pirke, K. M., \& Hellhammer, D. H. (1993). The "Trier Social Stress Test"- a tool for investigating psychobiological stress responses in a laboratory setting. Neuropsychobiology, 28, 76-81. doi: 119004

Kirschbaum, C., Wust, S., \& Hellhammer, D. (1992). Consistent sex differences in cortisol responses to psychological stress. Psychosomatic Medicine, 54, 648-657.

Kudielka, B. M., Hellhammer, D. H., \& Wust, S. (2009). Why do we respond so differently? Reviewing determinants of human salivary cortisol responses to challenge. Psychoneuroendocrinology, 34, 2-18. doi: 10.1016/j.psyneuen.2008.10.004

Kudielka, B. M., \& Kirschbaum, C. (2005). Sex differences in HPA axis responses to stress: A review. Biological Psychology, 69, 113-132. doi: 10.1016/j.biopsycho.2004.11.009

Kukolja, J., Thiel, C. M., Wolf, O. T., \& Fink, G. R. (2008). Increased cortisol levels in cognitively challenging situations are beneficial in young but not older subjects. Psychopharmacology, 201, 293-304. doi: 10.1007/s00213-008-1275-8

Lederbogen, F., Kirsch, P., Haddad, L., Streit, F., Tost, H., Schuch, P., . . . Meyer-Lindenberg, A. (2011). City living and urban upbringing affect neural social stress processing in humans. Nature, 474, 498-501. doi: 10.1038/Nature 10190

Lord, C., Steiner, M., Soares, C. N., Carew, C. L., \& Hall, G. B. (2012). Stress response in postpartum women with and without obsessivecompulsive symptoms: An fMRI study. Journal of Psychiatry \& Neuroscience, 37, 78-86. doi: 10.1503/Jpn.110005

Lovallo, W. (1975). The cold pressor test and autonomic function: A review and integration. Psychophysiology, 12, 268-282.

Lueken, U., Muehlhan, M., Evens, R., Wittchen, H. U., \& Kirschbaum, C. (2012). Within and between session changes in subjective and neuroendocrine stress parameters during magnetic resonance imaging: A controlled scanner training study. Psychoneuroendocrinology, 37, 12991308. doi: 10.1016/j.psyneuen.2012.01.003

Mitchell, L. A., MacDonald, R. A., \& Brodie, E. E. (2004). Temperature and the cold pressor test. The Journal of Pain, 5, 233-237. doi: 10.1016/ j.jpain.2004.03.004

Muehlhan, M., Lueken, U., Wittchen, H. U., \& Kirschbaum, C. (2011). The scanner as a stressor: Evidence from subjective and neuroendocrine stress parameters in the time course of a functional magnetic resonance imaging session. International Journal of Psychophysiology, 79, 118126. doi: 10.1016/j.ijpsycho.2010.09.009

Nater, U. M., Rohleder, N., Gaab, J., Berger, S., Jud, A., Kirschbaum, C., \& Ehlert, U. (2005). Human salivary alpha-amylase reactivity in a psychosocial stress paradigm. International Journal of Psychophysiology, 55, 333-342. doi: 10.1016/j.ijpsycho.2004.09.009

Nicolson, N. A. (2008). Measurement of cortisol. In L. J. Luecken \& L. C. Gallo (Eds.), Handbook of physiological research methods in health psychology (pp. 37-74). Los Angeles, CA: Sage.

Porcelli, A. J., Cruz, D., Wenberg, K., Patterson, M. D., Biswal, B. B., \& Rypma, B. (2008). The effects of acute stress on human prefrontal working memory systems. Physiology \& Behavior, 95, 282-289. doi: 10.1016/j.physbeh.2008.04.027

Pruessner, J. C., Declovic, K., Khalili-Mahani, N., Engert, V., Pruessner, M., Buss, C., . . L Lupien, S. (2008). Deactivation of the limbic system during acute psychosocial stress: Evidence from positron emission tomography and functional magnetic resonance imaging studies. Biological Psychiatry, 63, 234-240. doi: 10.1016/j.biopsych.2007. 04.041

Pruessner, J. C., Kirschbaum, C., Meinlschmid, G., \& Hellhammer, D. H. (2003). Two formulas for computation of the area under the curve represent measures of total hormone concentration versus timedependent change. Psychoneuroendocrinology, 28, 916-931.

Qin, S. Z., Hermans, E. J., van Marle, H. J. F., Luo, J., \& Fernandez, G. (2009). Acute psychological stress reduces working memory-related activity in the dorsolateral prefrontal cortex. Biological Psychiatry, 66, 25-32. doi: 10.1016/j.biopsych.2009.03.006

Rohleder, N., Nater, U. M., Wolf, J. M., Ehlert, U., \& Kirschbaum, C. (2004). Psychosocial stress-induced activation of salivary alphaamylase: An indicator of sympathetic activity? Annals of the New York Academy of Sciences, 1032, 258-263. doi: 10.1196/annals.1314. 033

Shuster, L. I., \& Lemieux, S. K. (2005). An fMRI investigation of covertly and overtly produced mono- and multisyllabic words. Brain Language, 93, 20-31. doi: 10.1016/j.band1.2004.07.007

Schwabe, L., Haddad, L., \& Schachinger, H. (2008). HPA axis activation by a socially evaluated cold-pressor test. Psychoneuroendocrinology, 33, 890-895. doi: 10.1016/j.psyneuen.2008.03.001 
Schwabe, L., \& Wolf, O. T. (2010). Stress impairs the reconsolidation of autobiographical memories. Neurobiology of Learning and Memory, 94, 153-157. doi: 10.1016/j.nlm.2010.05.001

Sinha, R., Lacadie, C., Skudlarski, P., \& Wexler, B. E. (2004). Neural circuits underlying emotional distress in humans. Annals of the New York Academy of Sciences, 1032, 254-257. doi: 10.1196/annals. 1314.032

Smeets, T., Cornelisse, S., Quaedflieg, C. W. E. M., Meyer, T., Jelicic, M., \& Merckelbach, H. (2012). Introducing the Maastricht Acute Stress Test (MAST): A quick and non-invasive approach to elicit robust autonomic and glucocorticoid stress responses. Psychoneuroendocrinology, 37, 1998-2008. doi: 10.1016/j.psyneuen.2012.04.012

Smeets, T., Jelicic, M., \& Merckelbach, H. (2006). Stress-induced cortisol responses, sex differences, and false recollections in a DRM paradigm. Biological Psychology, 72, 164-172. doi: 10.1016/j.biopsycho.2005. 09.004

Soliman, A., O’Driscoll, G. A., Pruessner, J., Joober, R., Ditto, B., Streicker, E., . . . Dagher, A. (2011). Limbic response to psychosocial stress in schizotypy: A functional magnetic resonance imaging study. Schizophrenia Research, 131, 184-191. doi: 10.1016/j.schres.2011.05.016

Tessner, K. D., Walker, E. F., Hochman, K., \& Hamann, S. (2006). Cortisol responses of healthy volunteers undergoing magnetic resonance imaging. Human Brain Mapping, 27, 889-895. doi: 10.1002/hbm. 20229

Ulrich-Lai, Y. M., \& Herman, J. P. (2009). Neural regulation of endocrine and autonomic stress responses. Nature Reviews Neuroscience, 10, 397-409. doi: 10.1038/Nrn2647
Van Cauter, E., \& Refetoff, S. (1985). Evidence for two subtypes of Cushing's disease based on the analysis of episodic cortisol secretion. The New England Journal of Medicine, 312, 1343-1349. doi: 10.1056/ NEJM198505233122102

van Marle, H. J. F., Hermans, E. J., Qin, S. Z., \& Fernandez, G. (2009). From specificity to sensitivity: How acute stress affects amygdala processing of biologically salient stimuli. Biological Psychiatry, 66, 649-655. doi: 10.1016/j.biopsych.2009.05.014

van Stegeren, A., Rohleder, N., Everaerd, W., \& Wolf, O. T. (2006). Salivary alpha amylase as marker for adrenergic activity during stress: Effect of betablockade. Psychoneuroendocrinology, 31, 137-141. doi: 10.1016/j.psyneuen.2005.05.012

Wang, J. J., Korczykowski, M., Rao, H. Y., Fan, Y., Pluta, J., Gur, R. C., . . . Detre, J. A. (2007). Gender difference in neural response to psychological stress. Social Cognitive and Affective Neuroscience, 2, 227-239. doi: 10.1093/Scan/Nsm018

Wang, J. J., Rao, H. Y., Wetmore, G. S., Furlan, P. M., Korczykowski, M., Dinges, D. F., \& Detre, J. A. (2005). Perfusion functional MRI reveals cerebral blood flow pattern under psychological stress. Proceedings of the National Academy of Sciences of the United States of America, 102, 17804-17809. doi: 10.1073/pnas.0503082102

Yang, H. Y., Zhou, Z. Y., Liu, Y., Ruan, Z. C., Gong, H., Luo, Q. M., \& Lu, Z. H. (2007). Gender difference in hemodynamic responses of prefrontal area to emotional stress by near-infrared spectroscopy. Behavioural Brain Research, 178, 172-176. doi: 10.1016/j.bbr.2006.11.039

(Received January 23, 2013; ACCEPTEd April 7, 2013) 